\title{
Luteolin exerts a marked antitumor effect in cMet-overexpressing patient-derived tumor xenograft models of gastric cancer
}

Jun Lu${ }^{1}$, Guangliang Li ${ }^{1}$, Kuifeng He${ }^{1}$, Weiqin Jiang ${ }^{1}$, Cong Xu', Zhongqi Li ${ }^{1}$, Haohao Wang ${ }^{1}$, Weibin Wang ${ }^{1}$, Haiyong Wang ${ }^{1}$, Xiaodong Teng ${ }^{2}$ and Lisong Teng ${ }^{1 *}$

\begin{abstract}
Background: Aberrated activation of cMet in gastric cancer contributes to tumor growth, angiogenesis and metastasis. cMet-overexpressing gastric cancer has a poor prognosis because of high tumor metastasis and limited therapeutic options. Luteolin is a common dietary flavonoid with antitumor properties. However, the antitumor effect of luteolin on cMet-overexpressing gastric cancer remain unclear.
\end{abstract}

Methods: Two cMet-overexpressing patient-derived human tumor xenograft (PDTX) models of gastric cancer were established, and treated with luteolin or vehicle to evaluate the antitumor effects of luteolin. Tumor specimens were subjected to H\&E staining and immunohistochemistry. MKN45 and SGC7901 cells that show high cMet expression were treated with varying concentrations of luteolin and evaluated by western blot, cell viability, apoptosis, migration, and invasion assays.

Results: Luteolin inhibited the tumor growth in cMet-overexpressing PDTX models. Immunohistochemistry demonstrated that expression of cMet, MMP9 and Ki-67 were significantly down-regulated. Luteolin inhibited proliferation, promoted apoptosis and reduced the invasiveness of MKN45 and SGC7901 cells. Western blot revealed that luteolin promoted the activation of apoptosis-related proteins, caspase-3 and PARP-1, and down-regulated the invasion-associated protein, MMP9. Further studies demonstrated that luteolin decreased the expression and phosphorylation of cMet, and downstream phosphorylation of Akt and ERK. In addition, luteolin down-regulated phosphorylated Akt independently of cMet. Blocking Akt and/or ERK with the PI3K inhibitor, LY294002, or the ERK inhibitor, PD98059, induced down-regulation of MMP9 and up-regulation of cleaved caspase-3 and PARP-1, resembling the effects of luteolin.

Conclusions: Our findings, for the first time, demonstrate that luteolin exerts marked antitumor effects in cMet-overexpressing PDTX models of gastric cancer, through a mechanism likely involving cMet/Akt/ERK signaling. These findings indicate that luteolin may act as a potential therapeutic option for cMet-overexpressing gastric cancer.

Keywords: Luteolin, cMet-overexpressing, Gastric cancer, Patient-derived tumor xenografts

\footnotetext{
* Correspondence: 11218203@zju.edu.cn

'Department of Surgical Oncology, The 1st Affiliated Hospital, School of Medicine, Zhejiang University, NO. 79 Qingchun Road, Hangzhou 310003, Zhejiang, China

Full list of author information is available at the end of the article
} 


\section{Introduction}

Gastric cancer (GC) is the one of the most commonly diagnosed cancers, and the second leading cause of cancer deaths worldwide [1,2]. Despite improvements in surgery and chemotherapy, the prognosis of advanced gastric cancer remains poor. cMet is a member of the receptor tyrosine kinase family, and the major signaling cascades activated by cMet include the phosphoinositide 3-kinase (PI3K)-Akt and Ras-mitogen-activated protein kinase (MAPK) pathways that are associated with tumor survival, growth, angiogenesis and metastasis $[3,4]$. cMet-overexpressing gastric cancer, which accounts for approximately $40 \%$ of all gastric cancer cases, has been shown to correlate with an advanced disease stage and poor prognosis [5-7]. Previous studies of gastric cancer have revealed that co-expression of hepatocyte growth factor (HGF) and c-Met has the potential to promote peritoneal dissemination, and that a high level of c-Met expression is involved in the mechanisms of liver metastasis $[3,8]$. Moreover, cMet-overexpressing gastric cancer cells can acquire resistance to therapy targeted against the HER family, such as epidermal growth factor receptor-2 (Her2) and the epidermal growth factor receptor (EGFR) $[9,10]$. cMet-overexpressing gastric cancer possesses a more aggressive cancer phenotype and has a poorer prognosis; therefore, optimizing drugs for the treatment of this type of gastric cancer is crucial.

Luteolin ( $3^{\prime}, 4^{\prime}, 5,7$-tetrahydroxyflavone) is one of the most common flavonoids found in various types of vegetables and fruits, such as celery, green peppers, carrots and olive oil. Luteolin shows strong anti-proliferative activity against a diversity of cancer cells, including breast, prostate and gastric cancers [11-13].

Previous studies have indicated that luteolin exerts its anti-tumor actions by affecting numerous biochemical pathways critical for the regulation of cell survival, apoptosis, angiogenesis and metastasis, including PI3K/Akt, nuclear factor- $\mathrm{kB}$ (NF- $\mathrm{kB})$, MAPKs, matrix metalloproteinases (MMPs) and E-cadherin [14-18]. In addition, recent experimental studies have shown that luteolin can suppress HGF-induced c-Met phosphorylation in HepG2 cells, and inhibit the expression of cMet in DU145 prostate cancer cells $[8,19]$. Although it has been suggested that luteolin possesses strong antitumor characteristics, an effect on cMet-overexpressing gastric cancer cells has yet to be clearly demonstrated.

One of the main obstacles hampering progress in oncological drug research is a lack of appropriate preclinical models. Patient-derived human tumor xenograft (PDTX) models, which closely retain the histopathologic, genetic and phenotypic features of the original clinical cancer, offer a powerful tool for the study of tumor biology and the evaluation of anticancer drugs.
Recently, we established PDTX models of colon carcinoma, and successfully evaluated a novel molecular drug $[20,21]$. In the present study, we evaluated the antitumor efficacy of luteolin in cMet-overexpressing PDTX models as well as in gastric cancer cell lines.

\section{Materials and methods \\ Reagents and drugs}

The antibodies against cMet, Akt and ERK, and phosphorylation-specific antibodies against phosphoMet (Y1234/1235), Akt (Ser308 and Ser473) and ERK (Thr202/Tyr204) were purchased from Cell Signaling Technology (Danvers, MA, USA). The antibodies against Her2, MMP9, Ki-67, caspase-3, cleaved caspase-3, poly (ADP-ribose) polymerase (PARP), cleaved PARP and glyceraldehyde 3-phosphate dehydrogenase (GAPDH) were obtained from Epitomics, Inc. (Burlingame, CA, USA). Horseradish peroxidase-conjugated secondary antibodies were sourced from Santa Cruz Biotechnology, Inc. (Santa Cruz, CA, USA). Luteolin was purchased from SigmaAldrich (St. Louis, MO, USA). LY294002 and PD98059 were obtained from Selleck Chemicals LLC (Houston, CA, USA).

\section{Establishment of xenografts and treatment protocol}

Four-to-six-week-old female BALB/c nude mice, purchased from Shanghai Slac Laboratory Animal Corporation (Shanghai, China), were housed with regular 12-hour light/ 12-hour dark cycles for at least three days before use. Animal care was carried out in accordance with the Principles of Laboratory Animal Care (NIH publication \#85-23, revised in 1985). All experimental protocols conducted in the present study were approved by the Institutional Animal Care and Use Committee of Zhejiang University (approval ID: SYXK[ZHE]2005-0072). Tumor specimens were obtained at initial surgery, after the patient had provided written informed consent. The patient had not received chemotherapy or radiation therapy before surgery. The tumors were diagnosed as poorly differentiated adenocarcinoma, according to WHO criteria (Additional file 1: Table S1). The PDTX xenograft models of gastric carcinoma were established as previously described [21,22]. Briefly, the tumors were implanted subcutaneously into the flanks of mice, under anesthesia with isoflurane. Growth of the xenografts was monitored at least twiceweekly by vernier caliper measurement of the length (a) and width (b) of the tumor. After reaching a volume of about $1500 \mathrm{~mm}^{3}$, the tumor was removed for serial transplantation.

Xenografts from the third generation (the second mouse-to-mouse passage) were used for the experiments, once the tumor volume had reached about $100 \mathrm{~mm}^{3}$. Mice with third generation xenografts were randomized into two groups (5-6 mice per group), to receive either 
luteolin $(10 \mathrm{mg} / \mathrm{kg})$ or dimethylsulfoxide (DMSO) vehicle by intraperitoneal (ip) injection daily for 1 month. Mouse weight and tumor volume were measured daily $(5$ mice per group). Tumor volume was calculated as (length $\times$ width $^{2}$ )/ 2. Relative tumor growth inhibition (TGI) was calculated using the formula: $\mathrm{TGI}=1-\mathrm{T} / \mathrm{C}$, where $\mathrm{T} / \mathrm{C}$ represents the relative tumor growth of luteolin-treated mice divided by the relative tumor growth of control (DMSO-treated) mice.

\section{Histology and immunohistochemistry}

Tumor-bearing mice were anesthetized and the tumors harvested. Tumor specimens were then fixed in $4 \%$ paraformaldehyde for 12 hours and embedded in paraffin. Five-micrometer sections were cut, dewaxed, rehydrated, and stained with hematoxylin and eosin (H\&E) as described previously [23]. For immunohistochemical staining, fivemicrometer sections were cut, dewaxed, rehydrated, and subjected to antigen retrieval. After quenching endogenous peroxidase activity and blocking nonspecific binding sites, the sections were incubated with primary antibodies against cMet (1:100), HER2 (1:100), MMP9 (1:200) and Ki-67 (1:500) at $4{ }^{\circ} \mathrm{C}$ for 12 hours. This was followed by a 30-min incubation with secondary antibody. Immunohistochemistry was performed using the streptavidin-biotin peroxidase complex method (Lab Vision, Fremont, CA, USA). The sections were observed using an optical microscope (Nikon, Tokyo Japan; 200x).

The expression of cMet was determined according to HercepTest guidelines, as follows: no membrane staining or membrane staining in $<10 \%$ of tumor cells, a score of 0 ; faint/barely perceptible partial membrane staining in $>10 \%$ of tumor cells, a score of $1+$; weak-to-moderate staining of the entire membrane in $>10 \%$ of tumor cells, a score of $2+$; and strong staining of the entire membrane in $>10 \%$ of tumor cells, a score of $3+$. Scores of 0 or $1+$ were considered as negative for MET overexpression, and scores of $2+$ or $3+$ were considered as positive [5]. For MMP9 assessment, we analyzed the staining intensity and the percentage of stained tumor cells. Staining intensity was scored as 0 (none), 1 (weak), 2 (moderate), and 3+ (strong), and the percentage was scored as $0 \%=0$ points, $\leq 25 \%=1$ point, 26 to $50 \%=2$ points, and $\geq 50 \%=$ 3 points. We calculated the final score by multiplying the respective scores. For Ki-67 assessment, one hundred cells were randomly selected and counted from five representative fields of each section, the percentage of stained tumor cells were calculated. All immunohistochemical slides were reviewed by two independent pathologists.

\section{Western blotting}

Briefly, lysates for immunoblotting were prepared by adding lysis buffer $(50 \mathrm{mM}$ Tris- $\mathrm{HCl}[\mathrm{pH} 7.4], 1 \%$ Nonidet P-40, $0.5 \%$ sodium deoxycholate, $150 \mathrm{mM}$
$\mathrm{NaCl}, 0.02 \%$ sodium azide, and $0.1 \%$ SDS) containing protease and phosphatase inhibitors (Sigma-Aldrich, St. Louis, MO, USA) to cells or tumor tissue homogenized in liquid nitrogen. Appropriate cell and tissue protein extracts were fractionated by SDS-PAGE and electro-transferred to polyvinylidene difluoride (PVDF) membranes (Millipore, Billerica, MA, USA). After blocking for $1 \mathrm{~h}$ at room temperature in 5\% milk in TBS-T (10 mM Tris- $\mathrm{HCl}$ [pH 7.5], $0.5 \mathrm{M} \mathrm{NaCl}$, and $0.05 \%$ [w/v] Tween 20), the membranes were incubated overnight at $4^{\circ} \mathrm{C}$ with appropriate primary antibodies. The next day, the membranes were washed and then incubated with suitable peroxidase-conjugated secondary antibodies for $1 \mathrm{~h}$ at room temperature. After washing three times with TBS, the blot was soaked for $1 \mathrm{~min}$ in $\mathrm{ECL}^{\mathrm{ma}}$ chemiluminescent detection reagents (Millipore, Billerica, MA, USA). The membranes were then placed between two sheets of plastic wrap and exposed to film (Kodak, Rochester, NY, USA) for $30 \mathrm{~s}$ in a darkroom. To show equal protein loading, the blots were stripped and reprobed for peroxidase-conjugated GAPDH antibody. The experiments were repeated at least three times.

\section{Cell culture}

MKN45, MKN28, BGC823, AGS and SGC7901 cells were obtained from the Culture Collection of the Chinese Academy of Sciences (Shanghai, China). These cell lines were passaged for fewer than 6 months after resuscitation. Cell lines were routinely cultured at $37^{\circ} \mathrm{C}$ in the presence of $5 \% \mathrm{CO}_{2}$ in RPMI 1640 (Invitrogen, Carlsbad, CA, USA) supplemented with 10\% FBS (Hyclone; GE Healthcare, Little Chalfont, UK).

\section{Cell viability assay}

The effect of luteolin on cell viability was assessed using the methyl-thiazolyl-tetrazolium (MTT) assay. In brief, cells were seeded into 96-well plates at 5000 cells per well. After overnight incubation, the cells were treated with DMSO vehicle $(1 \mu \mathrm{L} / \mathrm{mL})$ and varying concentrations of luteolin (20, 40 and $80 \mu \mathrm{M}$ in DMSO) for 24,48 and $72 \mathrm{~h}$. For measurement of cell growth, each well was incubated with MTT $(0.5 \mathrm{mg} / \mathrm{mL})$ for $4 \mathrm{~h}$ at $37^{\circ} \mathrm{C}$. Afterwards, the supernatant was removed and the formazan crystals dissolved in $200 \mu \mathrm{L}$ DMSO at room temperature. Absorbance of the solution was then measured at a 490-nm wavelength using an MRX II absorbance reader (Dynex Technologies, Chantilly, VA, USA).

\section{Cell apoptosis assay}

The apoptosis-inducing effect of luteolin was investigated using annexin V-fluorescein isothiocyanate (FITC) and flow cytometry. Cells grown in six-well plates were treated with varying concentrations of luteolin $(0-80 \mu \mathrm{M}$ in DMSO) for $24 \mathrm{~h}$. The cells were then harvested, washed 
twice in PBS, resuspended in binding buffer at a concentration of $1 \times 10^{6}$ cells $/ \mathrm{mL}$, and mixed with $5 \mu \mathrm{L}$ annexin V-FITC and $5 \mu \mathrm{L}$ propidium iodide for $15 \mathrm{~min}$. Stained cells were analyzed using an FC500 flow cytometer and CXP software (Beckman Coulter, Fullerton, CA, USA). The percentage of apoptotic cells was determined by the CXP software.

\section{In vitro migration and invasion assay}

Cell migration and invasion assays were carried out as described previously [24]. For these assays, the cells were pre-starved in serum-free medium for $12 \mathrm{~h}$. According to the protocol provided by the manufacturer (Millipore, Billerica, MA, USA), $900 \mu \mathrm{L}$ of medium with $10 \%$ FBS was added into the wells of a 24-well plate, and 8-mm pore transwell inserts were plated into these wells for 1-h rehydration at $37^{\circ} \mathrm{C}$. For the cell invasion assay, the upper chamber of a transwell was coated with Matrigel (BD Biosciences, San Jose, CA, USA) for $30 \mathrm{~min}$ at $37^{\circ} \mathrm{C}$ before rehydration. Starved cells were resuspended in serum-free medium at $3 \times 10^{5}$ cells per well for the invasion assay, and $1 \times 10^{5}$ cells per well for the migration assay, and seeded into the upper chamber. Both the upper chamber and lower chamber contained varying concentrations of luteolin $(0-20 \mu \mathrm{M}$ in DMSO). After incubation for $24 \mathrm{~h}$ at $37^{\circ} \mathrm{C}$, cells on the upper side of the membrane were removed with cotton swabs. The cells on the lower surface of the filters were fixed with methanol and stained with $0.1 \%$ crystal violet. The numbers of migrated or invaded cells were then counted from 5 random fields under $\times 200$ magnification.

\section{Statistical analysis}

All values were tested for normal distribution and are expressed as mean $\pm \mathrm{SD}$. Differences between groups were assessed using the Student's t-test (two-tailed). $\mathrm{P}<0.05$ was taken to indicate statistical significance. Statistical calculations were performed using SPSS 16.0 software (IBM Corporation, Armonk, NY, USA).

\section{Results}

\section{Histological and molecular characterization of the PDTX} models of gastric cancer

The PDTX models of gastric cancer were successfully established, with histological examinations of H\&E sections from the third generation xenografts of both Met-GC1 and Met-GC2 showing poorly differentiated adenocarcinoma consistent with the original clinical cancer (Figure 1a, b). We had previously ascertained that PDTX xenografts could retain the histopathologic and molecular characteristics of the original clinical cancer $[20,21]$. The expressions of cMet and HER2 were evaluated, since trastuzumab has been recommended as a treatment for patients with Her2-overexpressing gastric

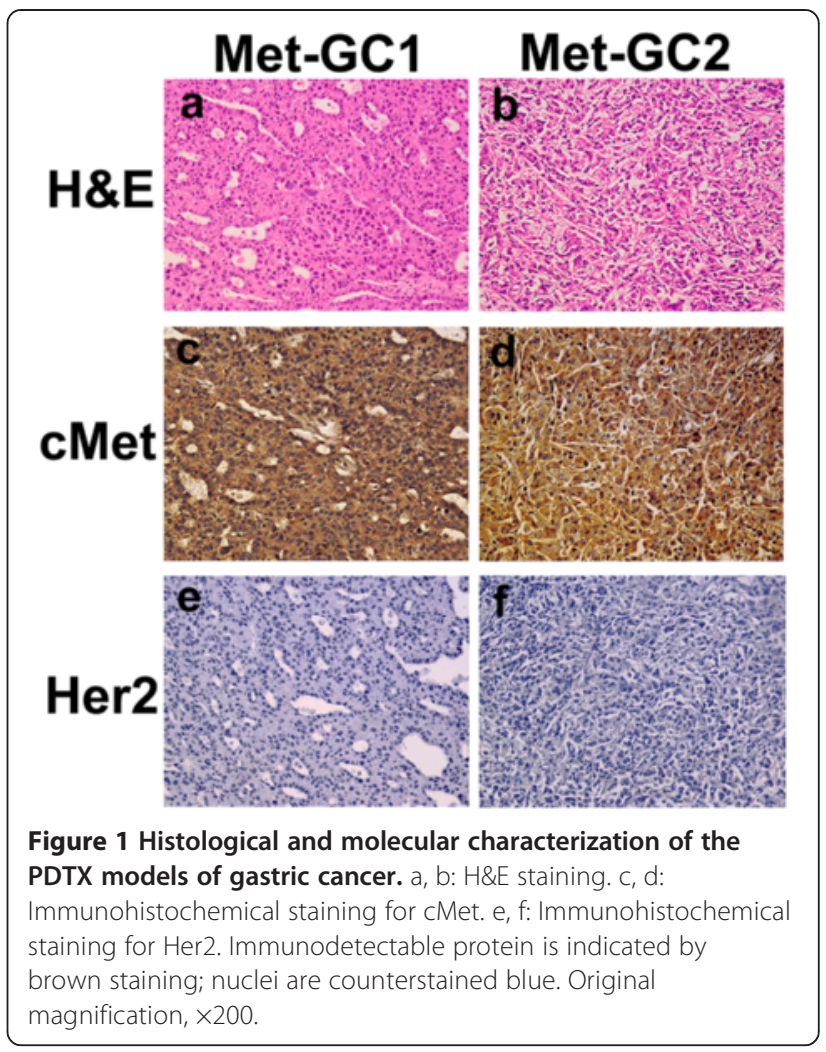

cancer. Immunohistochemical analysis showed that both the Met-GC1 and Met-GC2 xenograft models were positive for cMet overexpression $(+++)$ but negative for Her2 overexpression (-) (Figure 1c-f).

\section{Luteolin inhibits tumor growth in PDTX models of gastric cancer}

We next examined the effects of luteolin on growth of the PDTX models of gastric cancer. Luteolin significantly inhibited tumor growth, compared with the DMSO vehicle control, in both the cMet-GC1 and cMet-GC2 models (Figure 2a). No obvious differences were observed between the Met-GC1 and Met-GC2 models in the rate of tumor inhibition by luteolin (Figure 2b). No apparent toxicity or weight loss was observed with luteolin administration during the experimental period (Figure 2c).

Effects of luteolin administration on the expressions of cMet, p-cMet, Akt, p-Akt, ERK, p-ERK, MMP9 and Ki-67 in PDTX models of gastric cancer cMet-overexpression in gastric cancer is accompanied by abnormal activation of cMet signaling that contributes to tumor survival, growth and metastasis. We conducted immunohistochemical and Western blot analysis of the cMet signaling pathway. Immunohistochemistry demonstrated that the expression of cMet protein was significantly decreased in the luteolintreated groups. The expressions of MMP9, an indicator of potential metastatic capability, and Ki-67, a proliferation maker, were also greatly decreased in the luteolin-treated 

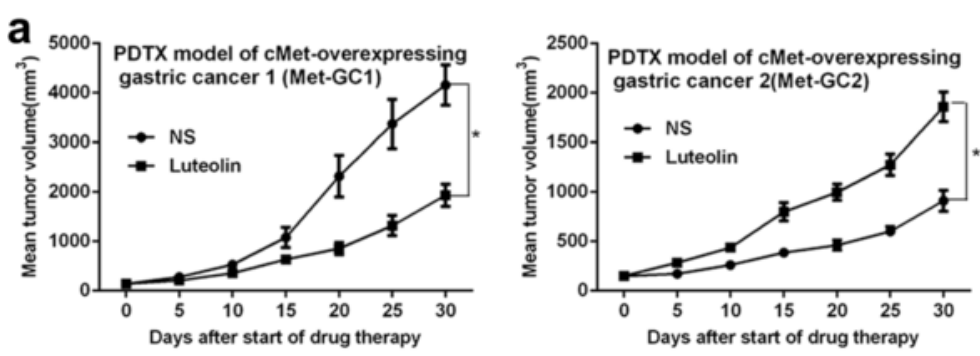

b
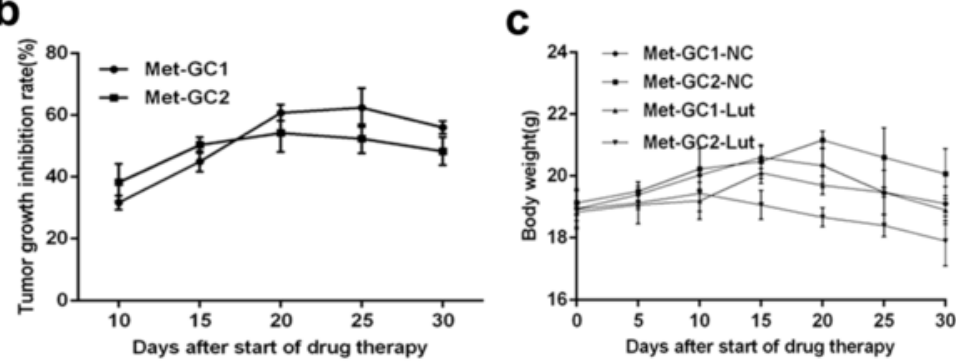

Figure 2 Luteolin inhibits tumor growth in cMet-overexpressing PDTX models of gastric cancer. a: Changes in tumor volume in Met-GC1 and Met-GC2 models of gastric cancer treated for 30 days with luteolin (10 mg/kg ip daily) or DMSO (as a vehicle control). b: Tumor growth inhibition rates in Met-GC1 and Met-GC2 models of gastric cancer treated with luteolin (10 mg/kg ip daily). c: Changes in mouse body weight in Met-GC1 and Met-GC2 models of gastric cancer treated for 30 days with luteolin (10 mg/kg ip daily) or DMSO (as a vehicle control). Data are presented as the mean $\pm \mathrm{SD}$. ${ }^{*} \mathrm{P}<0.05$ (Student's t-test).

groups (Figure 3, Additional file 2: Figure S1). Western blot analysis revealed down-regulated expression of cMet and phosphorylated cMet in the luteolin-treated groups. Only slight inhibition of phosphorylated ERK and Akt was observed in the luteolin-treated groups (Figure 4).

Luteolin inhibits proliferation and induces apoptosis of MKN45 and SGC7901 cells.To further investigate the possible mechanisms involved in the anti-tumor effects of luteolin in cMet-overexpressing gastric cancer, we first examined cMet expression in a series of gastric cancer cells, including MKN45, MKN28, AGS, BGC823 and SGC7901 cells. On the basis of these data, MKN45 and SGC7901 cells, which showed high expression of cMet, were selected for further study (Figure 5a). The MTT assay demonstrated that luteolin markedly inhibited the growth of MKN45 and SGC7901 gastric cancer cells in a concentration- and time-dependent manner (Figure 5b). To determine whether luteolin-induced cell death in MKN45 and SGC7901 cells was via an induction of apoptosis, we evaluated cell apoptosis using flow cytometry analysis, with propidium iodide and annexin- $\mathrm{V}$ staining. The data indicated that luteolin administration caused apoptosis in a concentration-dependent manner (Figure 5c). The percentage of apoptotic MKN45 and SGC7901 cells increased from $8.0 \%$ and $6.9 \%$, respectively, at $20 \mu \mathrm{M}$ luteolin to $23.7 \%$ and $20.2 \%$, respectively, at $80 \mu \mathrm{M}$ luteolin (Figure $5 \mathrm{~d}$ ). Western blot analysis revealed increased expression of cleaved caspase- 3 and cleaved PARP (indicators of apoptosis) (Figure 5e).
Luteolin inhibits the migration and invasion of MKN45 and SGC7901 cells.Low concentrations of luteolin $(0-20 \mu \mathrm{M})$ administered for $24 \mathrm{~h}$ did not affect the viability of MKN45 and SGC7901 cells. However, as shown in Figure 6b, administration of low concentrations of luteolin resulted in a concentration-dependent inhibition of migration and invasion of both MKN45 and SCG7901 cells. Since MMP9 expression was found to be decreased in Met-GC1 and Met-GC2 models treated with luteolin (see above), the expression of MMP9 was also evaluated in MKN45 and SGC7901 cells treated with luteolin for $24 \mathrm{~h}$. As shown in Figure 6c, luteolin also down-regulated MMP9 expression in both these gastric cancer cell lines. As MMP9 plays an important role in tumor invasion, the down-regulation of MMP9 may be involved in the inhibition of invasiveness by luteolin.

\section{Luteolin down-regulates cMet/Akt/ERK signaling in MKN45 and SGC7901 cells}

To further examine the involvement of the cMet signaling pathway in the anti-proliferative and anti-invasive actions of luteolin, we treated cells with various concentrations of luteolin $(0-80 \mu \mathrm{M})$ for $24 \mathrm{~h}$, and performed immunoblotting assays. As shown in Figure 7a, luteolin caused concentration-dependent reductions in total cMet protein and phosphorylated cMet levels in MKN45 and SGC7901 cells. Phosphorylated Akt and ERK, downstream signaling molecules of cMet, were also down-regulated. At a luteolin concentration of $20 \mu \mathrm{M}$, phosphorylated Akt 


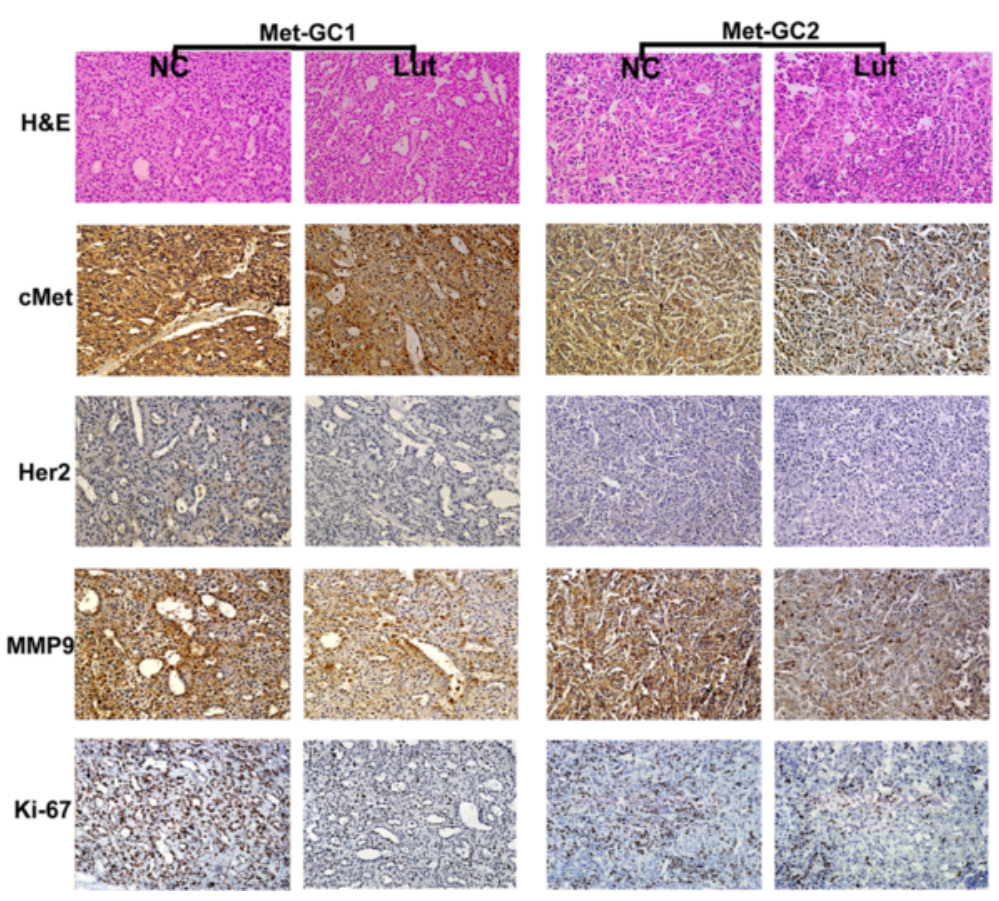

Figure 3 Effects of luteolin administration on the expressions of cMet, Akt, ERK, MMP9 and Ki-67 in PDTX models of gastric cancer. H\&E staining and immunohistochemistry were undertaken after administration for 30 days of either luteolin or DMSO (as a vehicle control). Immunodetectable protein is indicated by brown staining; nuclei are counterstained blue. Original magnification, $\times 200$.

was significantly down-regulated despite no obvious change in the phosphorylation of cMet (Figure 7a), indicting that Akt down-regulation preceded cMet down-regulation. To examine this hypothesis, Western blot analysis was carried out in MKN45 cells treated with luteolin for $6 \mathrm{~h}$, with reference to a previous study [19]. As shown in Figure 7b, at a concentration of $40 \mu \mathrm{M}$, luteolin significantly suppressed phosphorylated Akt but did not affect cMet phosphorylation. Furthermore, when AGS cells, which show low cMet expression, were treated with various concentrations of luteolin $(0-80 \mu \mathrm{M})$ for $24 \mathrm{~h}$, Western blot assays showed that luteolin significantly suppressed phosphorylated Akt with no obvious change in the phosphorylation of cMet and ERK (Figure 7c). Hence, the down-regulation of phosphorylated Akt appeared to be independent of cMet status, even occurring ahead of the change in cMet. Therefore, we further investigated whether the decrease in phosphorylated Akt led to the down-regulation of phosphorylated cMet. To do this, we used LY294002, a PI3K inhibitor, to determine whether prolonged inhibition of Akt phosphorylation resulted in a reduction in cMet phosphorylation. The data revealed that the administration of LY294002 to MKN45 cells for $24 \mathrm{~h}$ was without effect on cMet phosphorylation, despite an inhibition of phosphorylated Akt (Figure 7d). Further consideration of the data presented in Figure $7 \mathrm{a}, \mathrm{b}, \mathrm{c}$ suggested that the decrease in cMet expression mirrored the reduction in cMet phosphorylation. Thus, it was likely that the reduction in phosphorylated cMet was the result of a down-regulation in cMet expression. Together, the data indicate that luteolin could down-regulate total cMet and phosphorylated cMet, and inhibit the activity of downstream Akt and ERK signaling.

\section{Involvement of Akt and ERK in the regulation of caspase-3,} PARP-1 and MMP9

We next assessed the contributions of Akt and ERK in the effects of luteolin to induce apoptosis and inhibit invasiveness. MKN45 cells were harvested for Western blot analysis after exposure for $24 \mathrm{~h}$ to either the PI3K inhibitor, LY294002 $(20 \mu \mathrm{M})$, the ERK inhibitor, PD98059 $(50 \mu \mathrm{M})$, luteolin $(40 \mu \mathrm{M})$, or the combination of LY294002 and PD98059. As shown in Figure 7e, the administration of LY294002 or PD98059 inhibited p-Akt or $\mathrm{p}$-ERK, respectively. Combination treatment with LY294002 and PD98059 suppressed p-Akt and p-ERK simultaneously, similar to the effect of luteolin. Furthermore, the apoptosis-related proteins, cleaved caspase- 3 and cleaved PARP-1, were both up-regulated in all four treatment groups (LY294002, PD98059, luteolin, or LY294002 plus PD98059), while MMP9 was downregulated in all four treatment groups, and particularly in the LY294002 plus PD98059 group. Thus, both PD98059 and LY294002 were able to mimic the effects of luteolin on the regulation of caspase-3, PARP-1 and MMP9. Since caspase-3, PARP-1 and MMP9 are involved 


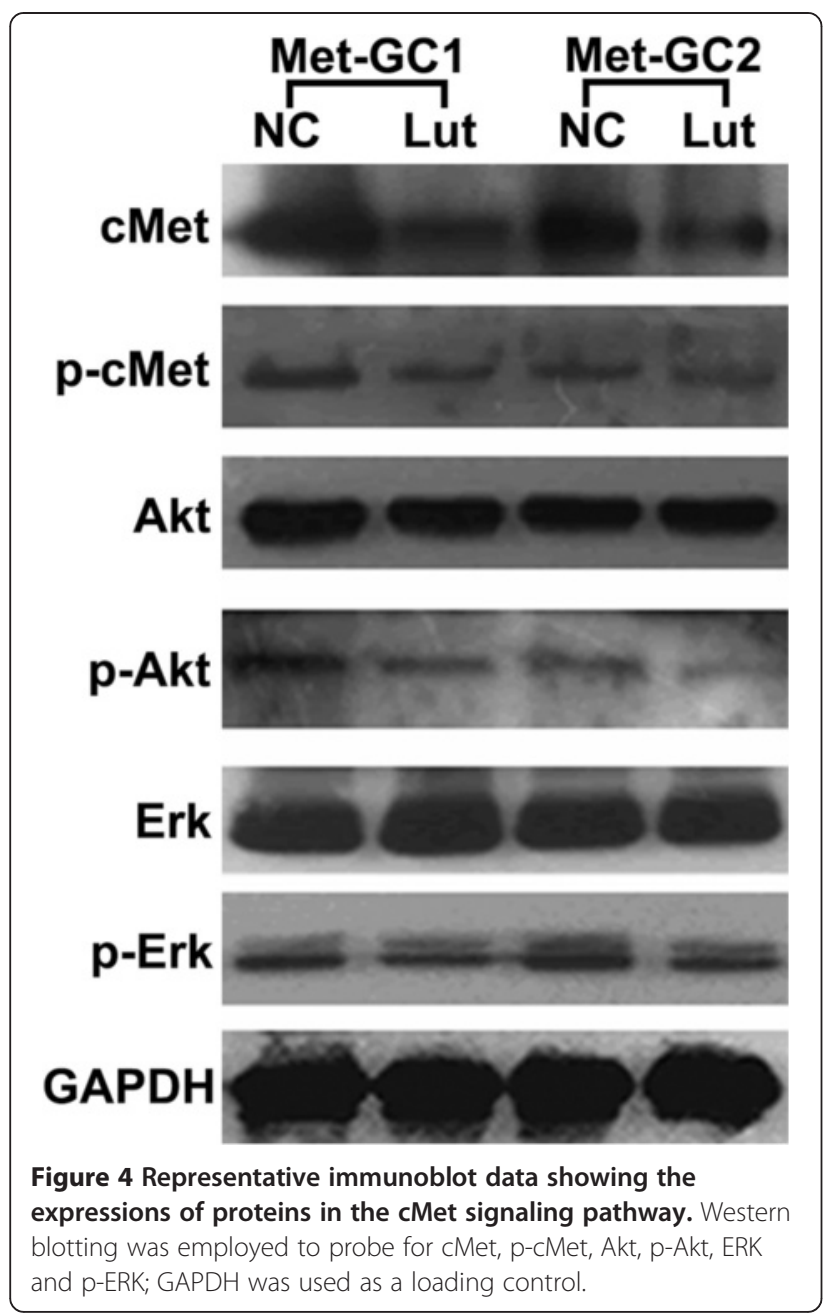

in cell apoptosis and invasion, we suggest that the downregulation of cMet/Akt/ERK is, at least in part, involved in the effects of luteolin to promote apoptosis and inhibit invasiveness.

\section{Discussion}

cMet-overexpressing gastric cancer is associated with advanced disease stage and poor prognosis [6,7]. The aggressive nature of cMet-overexpressing gastric cancer and the lack of effective therapeutic options make this cancer particularly clinically challenging, so it is crucial that new therapies are identified. However, research in this area has been hampered by a lack of clinically relevant experimental models. It is well established that PDTX models better retain the histopathologic, genetic and phenotypic features of the original tumor than conventional cell-implanted xenografts [22]. Hence, PDTX models have been increasingly used as a tool for the preclinical assessment of anticancer drugs. Our group has previously established PDTX models of colon carcinoma, and successfully evaluated a novel VEGF-targeted agent [20,21].
In this study, we evaluated the antitumor effects of luteolin in cMet-overexpressing PDTX models of gastric cancer. To our knowledge, this is the first study of luteolin in PDTX models. Our results showed that luteolin exerted a significant antitumor effect in these models of gastric cancer. An in vitro study further revealed that luteolin greatly inhibited the proliferative and invasive activity of MKN45 and SGC7901 gastric cancer cells, which highly expressed cMet. Immunohistochemical data demonstrated that luteolin greatly reduced the expressions of cMet, MMP9 and ki-67 (Figure 3); consistent with this, the in vitro study indicated that luteolin significantly down-regulated cMet signaling and MMP9 (Figures 6, 7). Together, these data suggest that luteolin may be a candidate therapeutic option for cMetoverexpressing gastric cancer.

Hyperactivation of the cMet signaling pathway has been frequently observed in cMet-overexpressing cancer, and reported to be associated with tumor survival, growth, angiogenesis and metastasis [3,4,7]. Previous studies have indicated that luteolin exerts its anti-tumor activity by affecting numerous biochemical pathways critical for the regulation of cell survival, apoptosis, angiogenesis and metastasis, including PI3K/Akt, NF-kB, MAPKs, MMPs and E-cadherin [14-18]. Lee et al. demonstrated that luteolin suppressed HGF-induced phosphorylation of c-Met in human hepatoma HepG2 cells. Coleman et al. further revealed that luteolin post-transcriptionally down-regulated c-Met expression independently of proteosomal/lysosomal degradation in DU145 prostate cancer cells. Recently, Wu et al. reported that luteolin can induce apoptosis by upregulating miR-34a in gastric cancer cells [25]. Anyway, further investigations were still needed to elucidate the possible mechanisms of antitumor effects of luteolin in gastric cancer. In the present study, we observed that luteolin decreased the expression and phosphorylation of cMet in both cMet-overexpressing gastric tumor tissue and gastric cancer cells with high cMet expression (Figures 3, 4, 7a). We further revealed that downstream Akt and ERK signaling was also down-regulated in MKN45 and SGC7901 cells. Interestingly, luteolin also caused a down-regulation of Akt without affecting the activity of cMet and ERK (Figure 7b, c), suggesting that the inhibition of Akt by luteolin could be independent of cMet. We also showed that luteolin-induced downregulation of phosphorylated Akt occurred ahead of the effects on cMet. We then confirmed that prolonged inhibition of phosphorylated Akt had no influence on phosphorylated cMet (Figure 7c). Further analysis of the results in Figure $7 \mathrm{a}, \mathrm{b}, \mathrm{c}$ suggested that the decrease in cMet expression mirrored the reduction in cMet phosphorylation. This finding implies that luteolin can down-regulate total cMet and phosphorylated cMet, and inhibit downstream Akt and ERK signaling, 


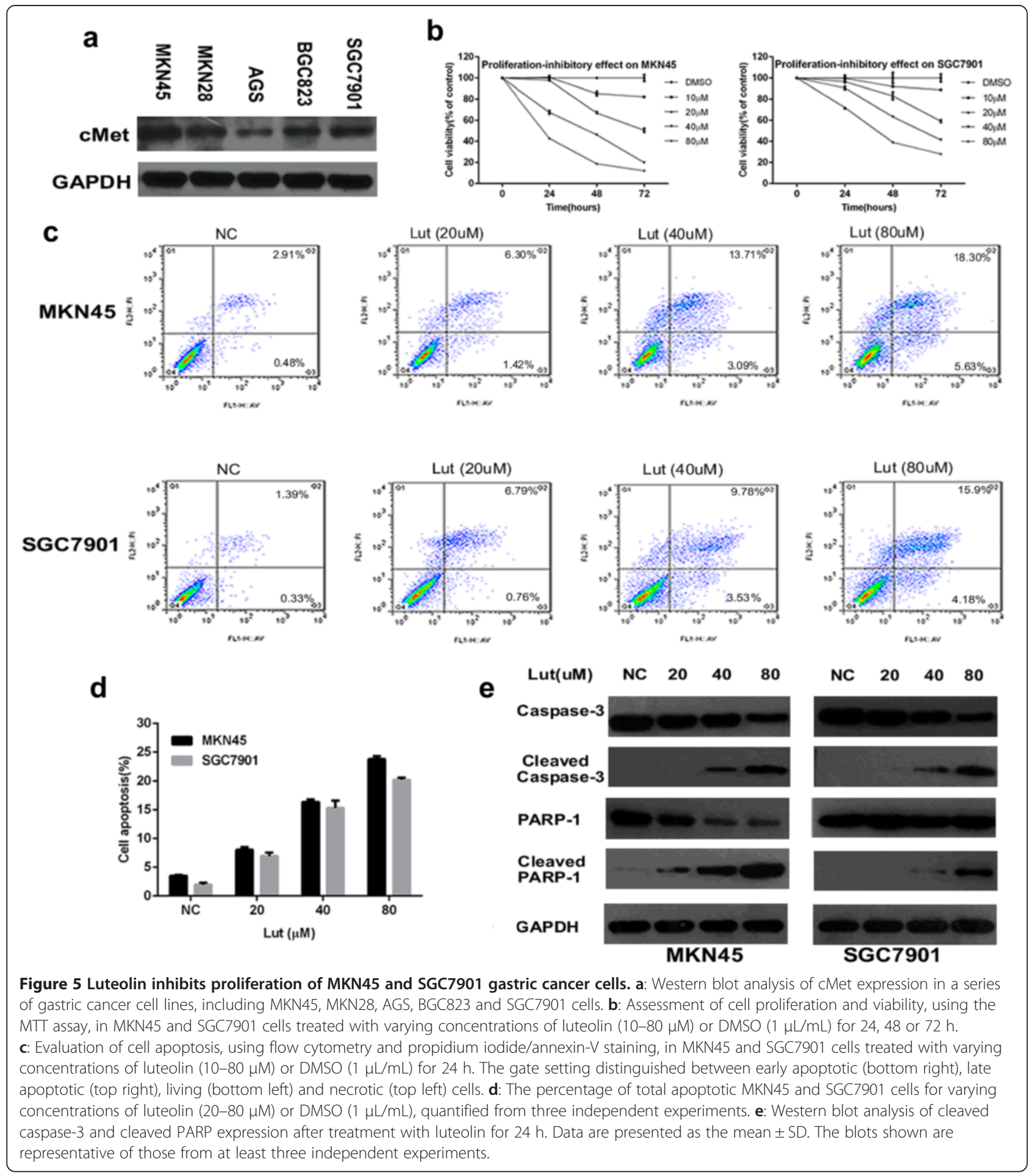

while also inhibiting Akt activity independently of cMet.

Previous research in different cell types has demonstrated that Akt and ERK signaling play a central role in the regulation of cell survival, proliferation and metastasis [26-28]. Activation of the Akt and ERK pathway is common in cMet-overexpressing cancer [4], and this activity can lead to a prevention of apoptosis $[29,30]$. In this study, we found that luteolin promoted the apoptosis of MKN45 and SGC7901 cells in a concentrationdependent manner, with activation of caspase-3 and PARP (Figure 5). As mentioned above, luteolin was able to 

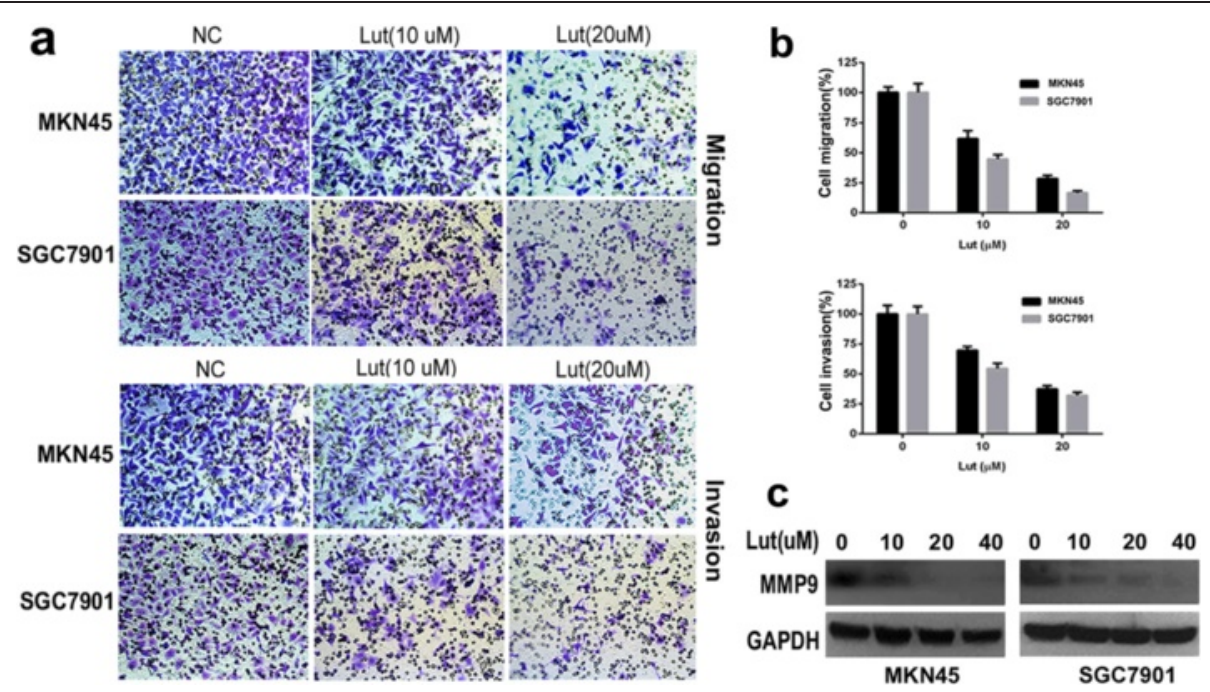

Figure 6 Inhibitory effects of luteolin on MKN45 and SGC7901 cell migration and invasion. (a) Representative images showing MKN45 and SGC7901 cell migration and invasion. Cells were seeded in transwell plates, incubated with 0-20 $\mu \mathrm{M}$ luteolin for $24 \mathrm{~h}$, and then fixed and stained with $0.1 \%$ crystal violet. (b) Cells (blue in color) on the lower surface were counted in triplicate, in three independent experiments, using a light microscope. (c) MMP9 expression evaluated by Western blotting. Data are presented as the mean \pm SD. Original magnification, $\times 200$.

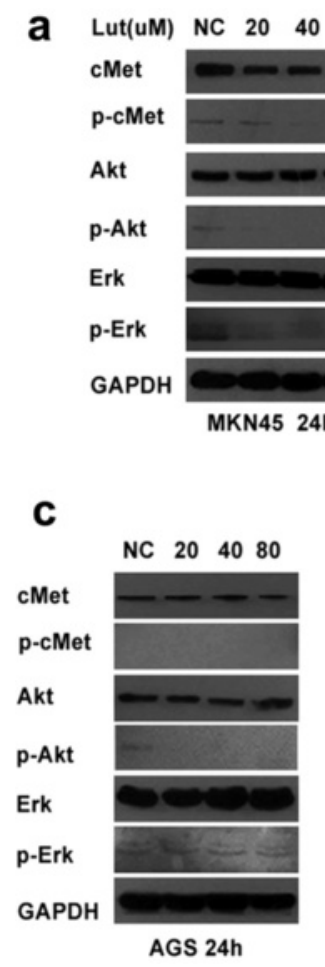

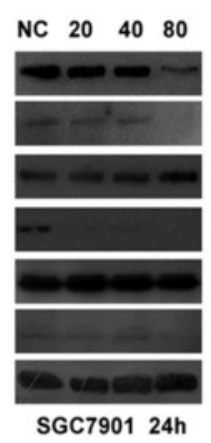

\section{d}

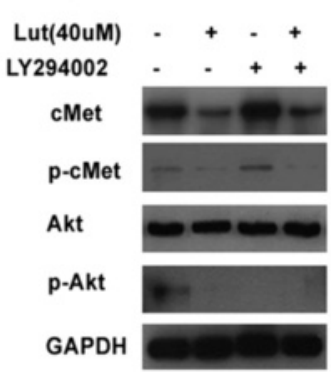

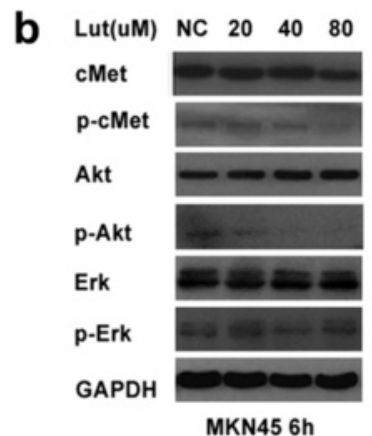

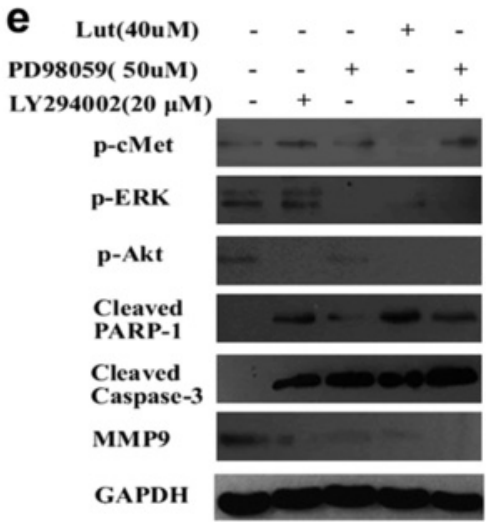

Figure 7 Effects of luteolin on cMet/Akt/ERK signaling pathways (a) Western blot analysis of MKN45 and SGC7901 cells treated with varying concentrations of luteolin $(\mathbf{0}-\mathbf{8 0} \mu \mathrm{M})$ for $\mathbf{2 4}$ h. (b) Western blot analysis of MKN45 cells treated with varying concentrations of luteolin $(0-80 \mu \mathrm{M})$ for 6 h. (c) Western blot analysis of AGS cells treated with varying concentrations of luteolin (0-80 $\mu \mathrm{M})$ for 24 h. (d) cMet/Akt/ERK status in MKN45 cells incubated for $24 \mathrm{~h}$ with LY294002 (a PI3K inhibitor; $20 \mu \mathrm{M}$ ), luteolin (40 $\mu \mathrm{M}$ ) or both. (e) Western blot analysis of the possible involvement of Akt/ERK in cell apoptosis and invasiveness. MKN45 cells were incubated for $24 \mathrm{~h}$ with either LY294002 (20 HM), PD98059 $(50 \mu \mathrm{M})$, luteolin $(40 \mu \mathrm{M})$, or a combination of LY294002 and PD98059. GAPDH was used as a loading control. The blots shown are representative of those from at least three independent experiments. 
down-regulate phosphorylated Akt and ERK. We further observed that both PD98059 (an ERK inhibitor) and LY294002 (an Akt inhibitor) could mimic the effects of luteolin on the activation of caspase- 3 and PARP-1. Previous investigations have also shown that LY294002 or PD98059 could inhibit the growth of gastric cancer cells and induce apoptosis [31-33]. Based on these findings, we suggest that luteolin may promote apoptosis partly via the down-regulation of phosphorylated Akt and ERK.

MMP9, which can degrade collagen IV, plays an important role in cancer metastasis [34]. It has been reported that MMP9 correlates with the invasion, metastasis and angiogenesis of gastric cancer [33,35]. Several studies have indicated that Akt and ERK regulate the expression of MMPs [35,36]. Previous research has demonstrated that the inhibition of Akt by LY294002 inhibited cancer cell invasion and down-regulated MMP9 expression [37,38]. In addition, blocking the ERK1,2 pathway with a selective chemical inhibitor, PD98059, could also down-regulate MMP9 [39]. In the present study, we found that MMP9 was down-regulated in luteolin-treated tumor tissue and cancer cells (Figures 3, 6c). Significant inhibitions of migration and invasion were also observed in the in vitro study (Figure 6). To determine whether down-regulation of phosphorylated Akt and ERK affected the expression of MMP9, we treated MKN45 and AGS cells with LY294002 and/or PD98059: we observed that LY294002 or PD98059 could down-regulate MMP9, an effect similar to that of luteolin (Figure 7d). Therefore, down-regulation of MMP9 may be involved in the inhibition of invasiveness by luteolin.

Previous investigations have also reported that luteolin can affect various receptors, such as EGFR, Her2 and the androgen receptor $[11,12,40]$. In the present study, we examined the cMet and HER2 status of the PDTX models. The expression of EGFR was also examined, and found to be Met-GC1 (-) and Met-GC2 (+) (immunohistochemistry; data not shown). However, the in vitro study only examined the cMet status of MKN45 and SGC7901 cells. In effect, there is a considerable amount of HER2 and EGFR expression in MKN45 and SGC7901 cells, so we cannot exclude crosstalk effects from other pathways. Moreover, we observed a decrease in phosphorylated Akt in luteolin-treated MKN45, SGC7901 and AGS cells. Although the present study has not definitively established the underlying mechanisms, a possible explanation is that luteolin can target Akt either directly or through other pathways. Further studies are merited to explore these possibilities. In addition, although we successfully established two cMet-overexpressing gastric cancer models in this study, we are now establishing more PDTX models, since it is essential that clinically reliable experimental systems are developed to facilitate the discovery of novel therapeutic options for cMetoverexpressing gastric cancer.

\section{Conclusions}

In summary, our findings strongly complement the current knowledge concerning the treatment of cMetoverexpressing gastric cancer. Our data demonstrate, for the first time, that luteolin exerts marked antitumor effects in cMet-overexpressing PDTX models of gastric cancer, at least in part via down-regulation of cMet/Akt/ERK signaling. These findings indicate that luteolin may act as a potential therapeutic option for cMet-overexpressing gastric cancer. Further study focusing on the role of luteolin in cMet-overexpressing gastric cancer will provide more detailed insights for the antitumor effects of luteolin in the future.

\section{Additional files}

Additional file 1: Table S1. Clinical characteristics of the patients used for developing PDTX models.

Additional file 2: Figure S1. Quantitative analysis of Ki-67 expressions after the administration of luteolin. Quantitative analysis of Ki-67 expression was determined as described in Materials and Methods. Data are presented as the means $\pm \mathrm{SD}$. ${ }^{*} \mathrm{P}<0.05$ (Student's t-test).

\section{Competing interest}

The authors declare that they have no competing interests.

\section{Authors' contributions}

$\mathrm{LT}$ and $J \mathrm{~L}$ participated in study design and coordination and drafted the manuscript. $J$, LT,GL, KH, WJ,CX performed the in vitro and in vivo experiments. ZL, HW, WW, XT completed immunochemistry staining. JL, HW analyzed the data. All authors read and approved the final manuscript.

\section{Acknowledgments}

This study was supported by the National Natural Science Foundation of China (No. 81272676), National Science and Technology Major Project of the Ministry of Science and Technology of China (No. 2013ZX09506015), Medical Science and Technology Project of Zhejiang Province (No.2011ZDA009).

\section{Author details}

${ }^{1}$ Department of Surgical Oncology, The 1st Affiliated Hospital, School of Medicine, Zhejiang University, NO. 79 Qingchun Road, Hangzhou 310003, Zhejiang, China. ${ }^{2}$ Department of Pathology, The 1st Affiliated Hospital, School of Medicine, Zhejiang University, NO. 79 Qingchun Road, Hangzhou 310003, Zhejiang, China.

Received: 11 June 2014 Accepted: 14 January 2015

Published online: 01 February 2015

\section{References}

1. Garcia M, Jemal A, Ward E, Center M, Hao Y, Siegel R, et al. Global cancer facts \& figures 2007, vol. 1. Atlanta, GA: American cancer society; 2007.

2. Jemal A, Bray F, Center MM, Ferlay J, Ward E, Forman D. Global cancer statistics. CA Cancer J Clin. 2011;61:69-90.

3. Bussolino F, Di Renzo MF, Ziche M, Bocchietto E, Olivero M, Naldini L, et al. Hepatocyte growth factor is a potent angiogenic factor which stimulates endothelial cell motility and growth. J Cell Biol. 1992;119:629-41.

4. Gherardi E, Birchmeier W, Birchmeier C, Vande Woude G. Targeting MET in cancer: rationale and progress. Nat Rev Cancer. 2012;12:89-103.

5. Lee HE, Kim MA, Lee HS, Jung EJ, Yang HK, Lee BL, et al. MET in gastric carcinomas: comparison between protein expression and gene copy number and impact on clinical outcome. Br J Cancer. 2012;107:325-33. 
6. Nakajima M, Sawada H, Yamada Y, Watanabe A, Tatsumi M, Yamashita J, et al. The prognostic significance of amplification and overexpression of c-met and c-erb B-2 in human gastric carcinomas. Cancer. 1999:85:1894-902.

7. Teng L, Lu J. CMET as a potential therapeutic target in gastric cancer (Review). Int J Mol Med. 2013;32:1247-54.

8. Lee WJ, Wu LF, Chen WK, Wang CJ, Tseng TH. Inhibitory effect of luteolin on hepatocyte growth factor/scatter factor-induced HepG2 cell invasion involving both MAPK/ERKs and PI3K-Akt pathways. Chem Biol Interact. 2006;160:123-33.

9. Chen CT, Kim H, Liska D, Gao S, Christensen JG, Weiser MR. MET activation mediates resistance to lapatinib inhibition of HER2-amplified gastric cancer cells. Mol Cancer Ther. 2012;11:660-9.

10. Kneissl J, Keller S, Lorber T, Heindl S, Keller G, Drexler I, et al. Association of amphiregulin with the cetuximab sensitivity of gastric cancer cell lines. Int J Oncol. 2012;41:733-44

11. Chiang CT, Way TD, Lin JK. Sensitizing HER2-overexpressing cancer cells to luteolin-induced apoptosis through suppressing p21(WAF1/CIP1) expression with rapamycin. Mol Cancer Ther. 2007;6:2127-38.

12. Chiu FL, Lin JK. Downregulation of androgen receptor expression by luteolin causes inhibition of cell proliferation and induction of apoptosis in human prostate cancer cells and xenografts. Prostate. 2008:68:61-71.

13. Wu B, Zhang Q, Shen W, Zhu J. Anti-proliferative and chemosensitizing effects of luteolin on human gastric cancer AGS cell line. Mol Cell Biochem. 2008;313:125-32.

14. Hong Z, Cao X, Li N, Zhang Y, Lan L, Zhou Y, et al. Luteolin is effective in the non-small cell lung cancer model with L858R/T790M EGF receptor mutation and erlotinib resistance. Br J Pharmacol. 2014;171:2842-53.

15. Lee EJ, Oh SY, Sung MK. Luteolin exerts anti-tumor activity through the suppression of epidermal growth factor receptor-mediated pathway in MDA-MB-231 ER-negative breast cancer cells. Food Chem Toxicol. 2012;50:4136-43

16. Ong CS, Zhou J, Ong CN, Shen HM. Luteolin induces G1 arrest in human nasopharyngeal carcinoma cells via the Akt-GSK-3beta-Cyclin D1 pathway. Cancer Lett. 2010;298:167-75.

17. Park SH, Kim JH, Lee DH, Kang JW, Song HH, Oh SR, et al. Luteolin 8-C-betafucopyranoside inhibits invasion and suppresses TPA-induced MMP-9 and IL-8 via ERK/AP-1 and ERK/NF-kappaB signaling in MCF-7 breast cancer cells. Biochimie. 2013:95:2082-90.

18. Zhou Q, Yan B, Hu X, Li XB, Zhang J, Fang J. Luteolin inhibits invasion of prostate cancer $\mathrm{PC} 3$ cells through E-cadherin. Mol Cancer Ther. 2009;8:1684-91.

19. Coleman DT, Bigelow R, Cardelli JA. Inhibition of fatty acid synthase by luteolin post-transcriptionally down-regulates c-Met expression independent of proteosomal/lysosomal degradation. Mol Cancer Ther. 2009;8:214-24.

20. Jin K, Lan $H_{1}$ Xie B, He K, Xu Z, Li G, et al. Antitumor effects of FP3 in combination with capecitabine on PDTT xenograft models of primary colon carcinoma and related lymphatic and hepatic metastases. Cancer Biol Ther. 2012;13:737-44.

21. Jin K, Li G, Cui B, Zhang J, Lan H, Han N, et al. Assessment of a novel VEGF targeted agent using patient-derived tumor tissue xenograft models of colon carcinoma with lymphatic and hepatic metastases. PLoS One. 2011;6:e28384

22. Tentler JJ, Tan AC, Weekes CD, Jimeno A, Leong S, Pitts TM, et al. Patient-derived tumour xenografts as models for oncology drug development. Nat Rev Clin Oncol. 2012:9:338-50

23. Wang Y, Xue H, Cutz JC, Bayani J, Mawji NR, Chen WG, et al. An orthotopic metastatic prostate cancer model in SCID mice via grafting of a transplantable human prostate tumor line. Lab Invest. 2005;85:1392-404.

24. Li G, Zhang J, Jin K, He K, Zheng Y, Xu X, et al. Estrogen receptor-alpha36 is involved in development of acquired tamoxifen resistance via regulating the growth status switch in breast cancer cells. Mol Oncol. 2013;7:611-24.

25. Wu H, Huang M, Liu Y, Shu Y, Liu P. Luteolin Induces Apoptosis by Up-regulating miR-34a in Human Gastric Cancer Cells. Technol Cancer Res Treat. Prepublished on November 26, 2014 as doi:10.7785/tcrt.2012.500434

26. Datta SR, Brunet A, Greenberg ME. Cellular survival: a play in three Akts. Genes Dev. 1999:13:2905-27.

27. Liu P, Cheng H, Roberts TM, Zhao JJ. Targeting the phosphoinositide 3-kinase pathway in cancer. Nat Rev Drug Discov. 2009:8:627-44.

28. Roberts PJ, Der CJ. Targeting the Raf-MEK-ERK mitogen-activated protein kinase cascade for the treatment of cancer. Oncogene. 2007:26:3291-310.
29. Cagnol S, Chambard JC. ERK and cell death: mechanisms of ERK-induced cell death-apoptosis, autophagy and senescence. FEBS J. 2010;277:2-21.

30. Franke TF, Hornik CP, Segev L, Shostak GA, Sugimoto C. PI3K/Akt and apoptosis: size matters. Oncogene. 2003:22:8983-98.

31. Chao X, Zao J, Xiao-Yi G, Li-Jun M, Tao S. Blocking of PI3K AKT induces apoptosis by its effect on NF-kappaB activity in gastric carcinoma cell line SGC7901. Biomed Pharmacother. 2010;64:600-4.

32. Choi IJ, Kim JS, Kim JM, Jung HC, Song IS. Effect of inhibition of extracellular signal-regulated kinase 1 and 2 pathway on apoptosis and bcl-2 expression in Helicobacter pylori-infected AGS cells. Infect Immun. 2003;71:830-7.

33. Shih PH, Yeh CT, Yen GC. Effects of anthocyanidin on the inhibition of proliferation and induction of apoptosis in human gastric adenocarcinoma cells. Food Chem Toxicol. 2005:43:1557-66.

34. Deryugina El, Quigley JP. Matrix metalloproteinases and tumor metastasis. Cancer Metastasis Rev. 2006;25:9-34.

35. Zheng H, Takahashi H, Murai Y, Cui Z, Nomoto K, Niwa H, et al. Expressions of MMP-2, MMP-9 and VEGF are closely linked to growth, invasion, metastasis and angiogenesis of gastric carcinoma. Anticancer Res. 2006;26:3579-83.

36. Kim D, Kim S, Koh H, Yoon SO, Chung AS, Cho KS, et al. Akt/PKB promotes cancer cell invasion via increased motility and metalloproteinase production. FASEB J. 2001;15:1953-62.

37. Ma J, Xie SL, Geng YJ, Jin S, Wang GY, Lv GY. In vitro regulation of hepatocellular carcinoma cell viability, apoptosis, invasion, and AEG-1 expression by LY294002. Clin Res Hepatol Gastroenterol. 2014;38:73-80.

38. Xing CG, Zhu BS, Fan XQ, Liu HH, Hou X, Zhao K, et al. Effects of LY294002 on the invasiveness of human gastric cancer in vivo in nude mice. World Gastroenterol. 2009;15:5044-52.

39. ZhenLing $W$, ZhongLiang H, BaoAn L. TGFB1 upregulates expression of MMP-2 and MMP-9 through ERK signaling pathway in gastric carcinoma cells. Cancer Research on Prevention and Treatment. 2009;36:91-4.

40. Lee LT, Huang YT, Hwang JJ, Lee PP, Ke FC, Nair MP, et al. Blockade of the epidermal growth factor receptor tyrosine kinase activity by quercetin and luteolin leads to growth inhibition and apoptosis of pancreatic tumor cells. Anticancer Res. 2002;22:1615-27.

\section{Submit your next manuscript to BioMed Central and take full advantage of:}

- Convenient online submission

- Thorough peer review

- No space constraints or color figure charges

- Immediate publication on acceptance

- Inclusion in PubMed, CAS, Scopus and Google Scholar

- Research which is freely available for redistribution 